\title{
Human collagen alpha-2 type I stimulates collagen synthesis, wound healing, and elastin production in normal human dermal fibroblasts (HDFs)
}

\author{
Su Jin Hwang ${ }^{1}$, Geun-Hyoung Ha ${ }^{1}$, Woo-Young Seo ${ }^{1}$, Chung Kwon Kim ${ }^{2}$, KyeongJin Kim ${ }^{3,4, *}$ E Sang Bae Lee ${ }^{5, *}$ \\ ${ }^{1}$ ABIOTECH Co., Ltd., Suwon $16675,{ }^{2}$ Department of Anatomy and Cell Biology, Biomedical Institute for Convergence at SKKU (BICS), \\ Sungkyunkwan University School of Medicine, Suwon 16419, ${ }^{3}$ Department of Biomedical Sciences, College of Medicine, Inha University, \\ Incheon 22212, ${ }^{4}$ Program in Biomedical Science \& Engineering, Inha University, Incheon 22212, ${ }^{5}$ Division of Life Sciences, Jeonbuk \\ National University, Jeonju 54896, Korea
}

\begin{abstract}
Skin aging appears to be the result of overlapping intrinsic (including genetic and hormonal factors) and extrinsic (extemal environment including chronic light exposure, chemicals, and toxins) processes. These factors cause decreases in the synthesis of collagen type I and elastin in fibroblasts and increases in the melanin in melanocytes. Collagen Type I is the most abundant type of collagen and is a major structural protein in human body tissues. In previous studies, many products containing collagen derived from land and marine animals as well as other sources have been used for a wide range of purposes in cosmetics and food. However, to our knowledge, the effects of human collagenderived peptides on improvements in skin condition have not been investigated. Here we isolate and identify the domain of a human COL1A2-derived protein which promotes fibroblast cell proliferation and collagen type I synthesis. This human COL 1A2-derived peptide enhances wound healing and elastin production. Finally, the human collagen alpha-2 type l-derived peptide (SMM) ameliorates collagen type I synthesis, cell proliferation, cell migration, and elastin synthesis, supporting a significant anti-wrinkle effect. Collectively, these results demonstrate that human collagen alpha-2 type I-derived peptides is practically accessible in both cosmetics and food, with the goal of improving skin condition. [BMB Reports 2020; 53(10): 539-544]
\end{abstract}

*Corresponding authors. Kyeongjin Kim, Tel: +82-32-860-9870; Fax: +82-32-885-8302; E-mail: kimkj@inha.ac.kr; Sang Bae Lee, Tel: +8263-270-4856; Fax: +82-63-270-3362; E-mail: leesb@jbnu.ac.kr

https://doi.org/10.5483/BMBRep.2020.53.10.120

Received 5 June 2020, Revised 16 June 2020, Accepted 20 July 2020

Keywords: Collagen synthesis, Elastin production, hCOL1A2, HDF, Wound healing

\section{INTRODUCTION}

Skin aging appears to be the result of overlapping intrinsic (including genetic and hormonal factors) and extrinsic (external environment including chronic light exposure, chemicals, and toxins) processes (1). These factors simultaneously lead to histopathological and immunohistochemical changes in each skin layer, as well as changes in skin appearance after UV exposure (2-4). Moreover, both intrinsic and extrinsic factors cause decreased synthesis of collagen type I and elastin in fibroblasts and increased melanin in melanocytes (5-7).

Collagen and elastin are required to maintain the structure of skin in the dermis, and also create its elasticity $(2,8)$. Interestingly, the collagen, the fibrous protein, plays a major role in supporting the mechanical strength of skin, and constitutes the majority of the dermis. The matured collagen is an alpha chain formed by three collagen strands. The fibrogenesis of collagen is associated with the repeated unique amino acid composition of glycine, proline, hydroxyproline, and alanine (9). The most abundant type of human collagen, collagen type $\mathrm{I}$, is a major structural protein which is prevalent in the fibrous connective tissues such as skin, bone, and tendon (9). The collagen type I is comprised by two identical alpha- 1 type I chains and one alpha-2 type I chain, which forms a triple helix (9).

Human dermal fibroblasts (HDFs) play vital roles in wound repair and tissue remodeling through cell proliferation, migration into the wound bed, synthesis of the extracellular matrix $(E C M)$, and expression of thick actin bundles in myofibroblasts (10). Previous studies indicated that growth factors/cytokines such as fibroblast growth factors (FGF), transforming growth factor- $\beta 1$ (TGF- $\beta 1$ ), and epidermal growth factor (EGF) directly or indirectly affect HDF motility (10-12). The effects of this growth hormone cover a broad range of biological phenotypes including regeneration, cell proliferation, and metabolism (13-15).

In previous studies, many products containing collagen or denatured forms of collagen derived from land animals, marine animals or other sources have been used for a wide range of purposes in cosmetics and food. There is controversy over the

ISSN: 1976-670X (electronic edition)

Copyright (c) 2020 by the The Korean Society for Biochemistry and Molecular Biology

(ㄷ) This is an open-access article distributed under the terms of the Creative Commons Attribution Non-Commercial License (http://creativecommons.org/licenses/by-nc/4.0) which permits unrestricted non-commercial use, distribution, and reproduction in any medium, provided the original work is properly cited. 
effects of collagen derived from food or supplements because oral intake of native collagen or its partially hydrolyzed form, gelatin, is not efficiently absorbed in the body (16). However, several evidences have shown beneficial effects of small collagen-derived peptides, which show higher absorbability compared to native collagen in a wide variety of tissues including skin, bone, and muscle in humans (16-21). Moreover, collagen has been isolated from many marine organisms including fish and others (22-24). Collagen-derived peptides from aquatic sources, such as rainbow trout skin, have unique biological properties with respect to antioxidant and anti-skin aging activity when compared to collagen peptides derived from land animals. Moreover, collagen-derived peptides from sea and freshwater rainbow trout skin had similar amino acid compositions and molecular weight (25-27). However, the effects of human collagen-derived peptides on improvements in skin condition have not been investigated. In this study, we found that a human-derived collagen peptide improves skin condition, supporting the practical applicability of this peptide in cosmetic and food compositions.

\section{RESULTS AND DISCUSSION}

\section{Stimulatory effect of hCOL1A2 domains on fibroblast cell} proliferation and collagen type I synthesis.

It has previously been reported that collagen type I synthesis and fibroblast cell proliferation are required to maintain the elasticity and strength of skin (28-30). To address the biologi-

(A)

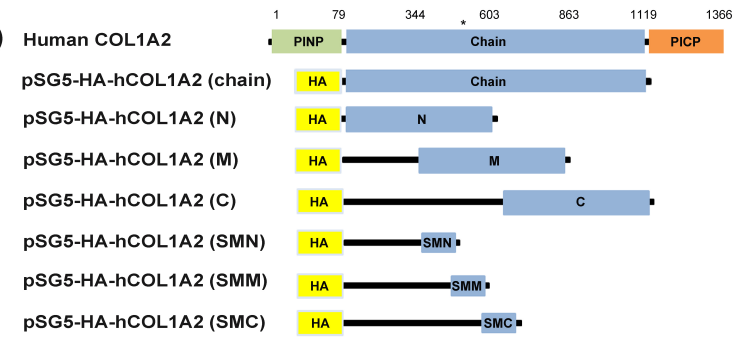

(B)

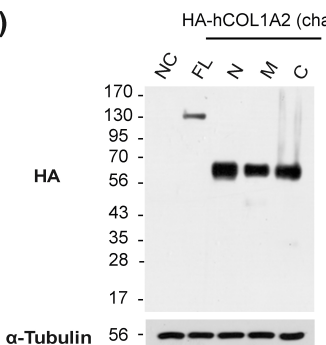

(C)

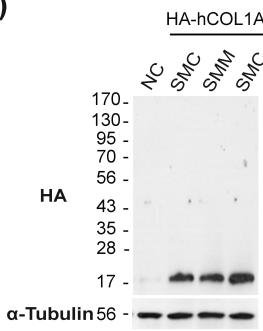

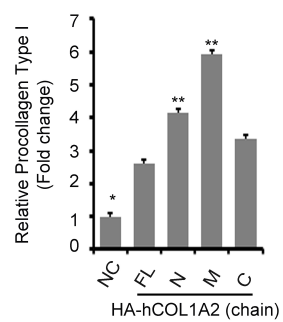

(D)
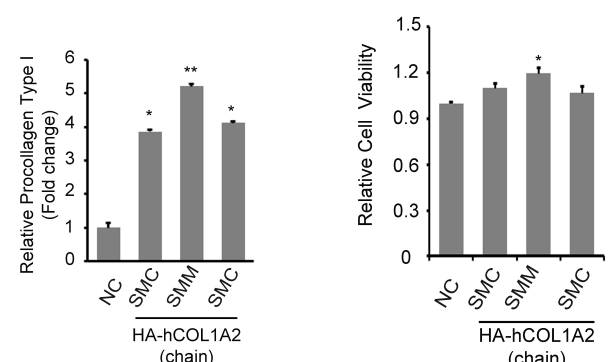

Fig. 1. Defining the domains of Human COL1A2 (hCOL1A2) involved in collagen synthesis. (A) Diagrams of Human full-length COL1A2 (hCOL1A2) and various deletion mutants. (B) HDF cells were transfected with plasmids encoding HA, HA-tagged COL1A2 FL, and each of the deletion mutants (N, M, and C). $48 \mathrm{~h}$ after transfection, cells were collected and cell lysates were analyzed by immunoblotting with anti-HA and anti-tubulin antibodies (left panel). The amount of collagen Type I was measured with an ELISA kit in transfected HDF cultured media (right panel). (C, D) HDF cells were transfected with plasmids encoding HA and each of the indicated mutants (SMN, SMM, and SMC). 48 $\mathrm{h}$ after transfection, cells were collected and cell lysates were analyzed by immunoblotting with anti-HA and anti-tubulin antibodies (left panel). The amount of collagen Type I was measured with an ELISA kit in transfected HDF cultured media (right panel). (D) Cell proliferation, which was induced by transfection with plasmids encoding HA and each of the indicated mutants (SMN, SMM, and SMC), was determined by MTT assay. Results are presented as mean \pm SEM of three independent experiments. The student's $t$-test was used for statistical analyses $(* P<0.05, * * P<0.005)$. 
cal function of human alpha-2 type I collagen (hCOL1A2) in skin fibroblasts, we examined the effects of hCOL1A2 domains on collagen type I synthesis and fibroblast cell proliferation. First, we generated a human COL1A2 (hCOL1A2) full-length (chain) and various deletion mutants (Fig. 1A). The effects of collagen type I synthesis were examined in HDF cells transfected with plasmids encoding HA, HA-tagged COL1A2 FL (chain domain), and each of the deletion mutants $(\mathrm{N}, \mathrm{M}$, and C). Cells were analyzed $48 \mathrm{~h}$ post-transfection by immunoblotting and the amount of collagen Type I was measured with an ELISA kit. The M domain of the COL1A2 chain significantly increased collagen type I synthesis compared to HA, COL1A2 FL (chain domain), and other deletion mutants (Fig. 1B). In addition, HDF cells were transfected with plasmids encoding HA and each of the indicated mutants (SMN, SMM, and SMC). Cells were analyzed $48 \mathrm{~h}$ post-transfection by immunoblotting and the amount of collagen Type I was measured with an ELISA kit. Collagen type I synthesis was significantly increased by treatment with the SMM domain of the COL1A2 chain compared to plasmids encoding $\mathrm{HA}$ and others mutants (SMN and SMC) (Fig. 1C). Further, the SMM domain of the COL1A2 chain increased fibroblast proliferation, compared to plasmids encoding $\mathrm{HA}$ and other mutants (Fig. 1D). These data indicate that the hCOL1A2-derived peptide domain (SMM) significantly stimulates collagen synthesis and fibroblast proliferation.

\section{Isolation and identification of recombinant hCOL1A2 (SMM) protein}

We generated a His-tagged hCOL1A2 (SMM) and cloned it into the pET-28a (His-tag) vector. Recombinant proteins were overexpressed in the E. coli Rosetta2 (DE3) strain and purified as previously described (31). To determine the molecular composition ratio, the purified hCOL1A2 (SMM) was analyzed by SDS-PAGE. Purified recombinant hCOL1A2 (SMM) was eluted and visualized as a single band around $\sim 20 \mathrm{kDa}$ (Fig. 2A). Isolated hCOL1A2 (SMM) was identified using an anti-His antibody (Fig. 2B) and was confirmed with an anti-COL1A2 (SMM-specific) antibody using western blot analysis (Fig. 2C).

To confirm whether the protein was actually purified recombinant hCOL1A2 (SMC), we performed in an in-gel digest with trypsin and analyzed the peptides by liquid chromatographymass spectrometry (LC/MS). Fig. 2D and Supplementary Table 1 show a representative MS/MS spectrum for the identified peptides, which originated from hCOL1A2 $\left({ }^{554}\right.$ GEQGPPGPPGFQGLPGP SGPAGEVGKPGER ${ }^{573}, 2810.373 \mathrm{~m} / \mathrm{z}$ ) and hCOL1A2 with Hyp residues $\left({ }^{465}\right.$ EGpVGLpGIDGRPGpIGpAGAR ${ }^{486}, 2104.044$ m/z).

\section{The collagen biosynthesis and cell proliferation effects of recombinant hCOL1A2 (SMM) protein}

A previous study reported that TGF- $\beta 1$ significantly increases collagen type I synthesis and proliferation in embryonic pulmonary fibroblasts and human dermal fibroblast (HDF) cells in crowded culture $(7,32)$. To investigate the effect of collagen synthesis, we determined the amount of secreted collagen type $I$ in the culture media of HDF cells stimulated with TGF- $\beta 1$ or hCOL1A2 (SMM) proteins (1, 10, and $100 \mu \mathrm{g} / \mathrm{ml})$. Collagen type I synthesis was increased by treatment with hCOL1A2 (SMM) protein in a dose-dependent manner (Fig. 3A). In addition, treat-
(A)

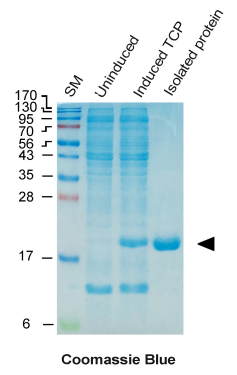

(D)

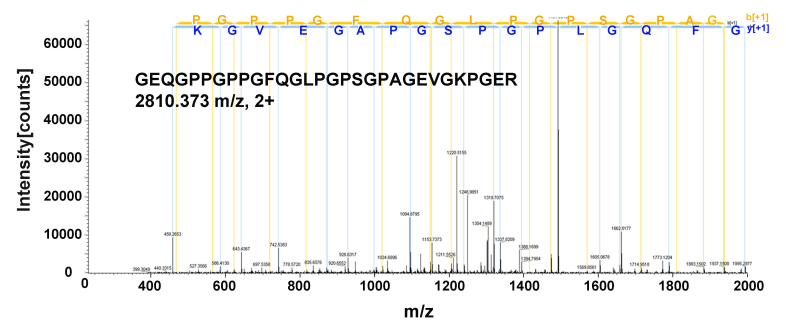

Fig. 2. Identification of purified hCOL1A2 (SMM) by SDS-PAGE and Western blot analysis. (A) SDS-PAGE showing isolated hCOL1A2 (SMM) with a prominent band; arrows indicate that hCOL1A2 (SMM) was identified using (B) anti-His and (C) anti-hCOL1A2 antibodies by western blot analysis. (D) Identification of hCOL1A2 (SMM) protein in the gel band of isolated hCOL1A2 (SMM) by LC-MS/MS analysis. The panels show the representative MS/MS spectrum for the identified peptides of GEQGPPGPPGFQGLPGPSGPAGEVGKPGER $(2810.373 \mathrm{~m} / \mathrm{z},+2)$. 
ment with hCOL1A2 (SMM) protein induced fibroblast proliferation in a dose-dependent manner (Fig. 3B). Next, we compared the effects of epidermal growth factor (EGF), which has previously been reported to increase collagen type I synthesis and HDF cell proliferation (33). hCOL1A2 (SMM) protein (at $1 \mu \mathrm{g} / \mathrm{mL}$ ) and $0.1 \mu \mathrm{g} / \mathrm{ml}$ EGF showed similar activity for the induction of collagen type I synthesis (Fig. 3C) and fibroblast cell proliferation (Fig. 3D). Together, these results demonstrated that hCOL1A2 (SMM) significantly increased collagen type I synthesis and fibroblast proliferation. Further, hCOL1A2derived protein $(\mathrm{SMN})$ was detectable in HDF cell lysates after washing out the culture medium containing hCOL1A2-derived
(A)

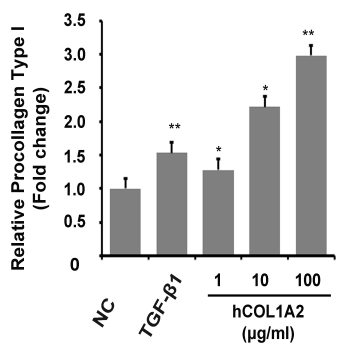

(C)

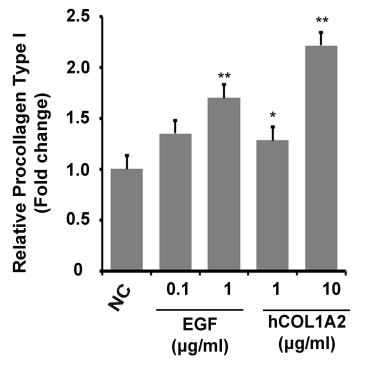

(B)

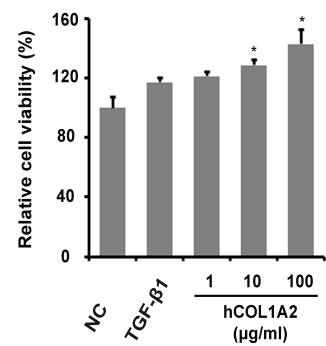

(D)

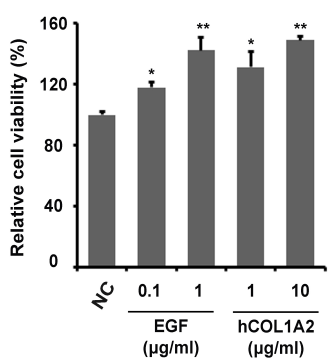

Fig. 3. hCOL1A2 (SMM) stimulates collagen synthesis and cell proliferation in primary human dermal fibroblast (HDF) cells. (A) HDF cells were treated with hCOL1A2 (SMM) $(1,10,100 \mu \mathrm{g} / \mathrm{ml})$ and TGF- $\beta 1(5 \mathrm{ng} / \mathrm{ml})$ for $48 \mathrm{~h}$. The amount of collagen Type I was measured with an ELISA kit in HDF cultured media. (B) HDF cells were treated with hCOL1A2 (SMM) $(1,10,100 \mu \mathrm{g} / \mathrm{ml})$ and TGF- $\beta 1$ (5 $\mathrm{ng} / \mathrm{ml})$ for $48 \mathrm{~h}$. Cell proliferation was determined by MTT assay. (C, D) HDF cells were treated with EGF $(0.1,1 \mu \mathrm{g} / \mathrm{ml})$ and hCOL1A2 (SMM) (1, 10 $\mu \mathrm{g} / \mathrm{ml}$ ) for $48 \mathrm{~h}$. The amount of collagen Type I was measured by ELISA in HDF cultured media (C). Cell proliferation, which was induced by EGF and hCOL1A2 (SMM), was determined by MTT assay (D). Results are mean \pm SEM of three independent experiments. A student's $t$-test was used for statistical analyses $(* P<0.05, * * P<0.005)$.

(A)

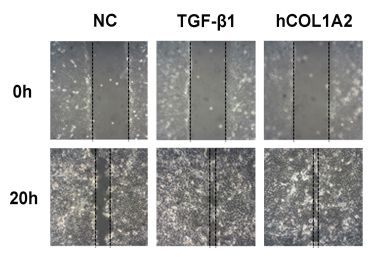

(B)

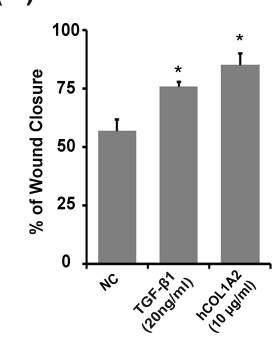

(C)

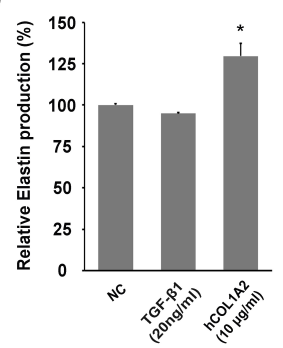

(D)

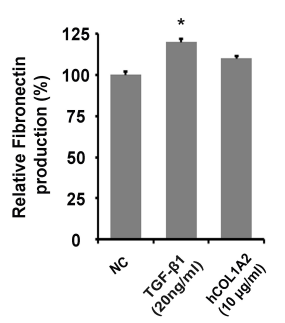

Fig. 4. Human COL1A2-derived protein increased wound healing efficiency and elastin production, but not fibronectin production. (A) $\mathrm{HaCaT}$ cells were grown to confluence on six-well tissue culture plates. Cells were serum-starved for 12 hours. Cells treated with TGF- $\beta 1$ $(20 \mathrm{ng} / \mathrm{ml})$ and hCOL1A2 $(10 \mu \mathrm{g} / \mathrm{ml})$ were then used in an in vitro wound assay. Migration was monitored for up to 20 hours. The same fields were photographed immediately after wounding and 20 hours later, and pictures were superimposed using Adobe Photoshop. Areas were measured using Image J. A representative result is shown. (B) Cell migration was monitored for up to 20 hours and quantified by image analysis. Results are presented as the mean \pm SEM of three independent experiments. The student's $t$-test was used for statistical analyses $(* \mathrm{P}<0.05)$. (C, D) HDF cells were treated with hCOL1A2 $(\mathrm{SMM})(10 \mu \mathrm{g} / \mathrm{ml})$ and TGF- $\beta 1(20 \mathrm{ng} / \mathrm{ml})$ for $48 \mathrm{~h}$. The amount of elastin (C) and fibronectin (D) were measured by ELISA in HDF cultured media. Results are presented as the mean \pm SEM of three independent experiments. The student's t-test was used for statistical analyses $(* P<0.05)(41)$. 
protein (SMN) (Supplementary Fig. 1), suggesting that SMN is bioactive, similar to hydrolyzed collagen peptides, and has benefits in absorption and utilization.

\section{In vitro scratch-wound healing assay and Elastin production} Previous studies suggested that the abundant amino acid residues in collagen peptides serve as additional nutrients for cellular growth and proliferation (22). To evaluate the effects of hCOL1A2 (SMM) on cell adhesion and growth, we utilized the human keratinocyte cell line $\mathrm{HaCaT}$, which has previously been utilized in in vitro skin wound healing models (34). We investigated the effect of TGF- $\beta 1$ and hCOL1A2 (SMM) on wound closure in $\mathrm{HaCaT}$ cell monolayers. As shown in Fig. 4A and $4 \mathrm{~B}$, scratch closure occurred more quickly in the presence of hCOL1A2 (SMM) compared to TGF- $\beta 1$ and the negative control (NC). We examined the wound closure assay and statistically analyzed the data which derived from captured images at least five field in three independent experiments (Fig. 4B). At 20 hours of incubation, the wound closure rate of the cells showed 75$90 \%$ by hCOL1A2 (SMM) treatment, while the control group showed less than $60 \%$.

A previous study demonstrated that skin is subject to intrinsic and extrinsic aging, which are both associated with histopathological and immunohistochemical changes (1). Such a skin aging process may include qualitative and quantitative changes, including diminished or defective collagen and elastin synthesis in the dermis $(35,36)$. To examine the effect on elastin synthesis, we quantified elastin in the culture media of HDF cells stimulated with TGF- $\beta 1$ or hCOL1A2 (SMM) proteins $(10 \mu \mathrm{g} / \mathrm{ml})$. Elastin synthesis was significantly increased with hCOL1A2 (SMM) protein treatment compared to TGF- $\beta 1$ and the negative control (NC, media treatment only) whereas fibronectin synthesis was not affected by hCOL1A2 (SMM) (Fig. 4C and 4D). Together, these results demonstrate that a collagen peptide (SMM) from human collagen alpha-2 Type I induces cell migration and elastin synthesis.

According to previous study, collagen peptides inhibited the expression of metalloproteinases (MMP1 and MMP3) release while simultaneously increasing elastin synthesis $(7,37)$. Matrix metalloproteinases (MMP1 and MMP3), the major collagenases in human, which were induced by ultraviolet irradiation (UV)/ sun exposure and natural skin aging, mediate the degradation of fibrillar type I collagen and elastic fiber (38-40). In contrast, treatment of the collagen peptides or derivatives such as a hCOL1A2 (SMM) provides the anti-skin aging effect through collagen expression/activation (collagen chain trimerization) and activation of TGF- $\beta$ signal pathway (37). In conclusion, the human collagen alpha-2 type I-derived peptide (SMM) enhances collagen type I and elastin synthesis, cell proliferation, and cell migration, supporting a significant anti-wrinkle effect. Additionally, hCOL1A2 (SMM) might be induced by relating hydroxylases such as previously developed collagen derivatives to supporting the anti-skin aging effect. The hCOL1A2 (SMM) induces anti-wrinkle effects, including proliferation of fibroblast and wound healing, but it has not been determined whether SMM can be used as a cosmetic ingredient. Therefore, comparing the functionality of hCOL1A2 (SMM) with the existing functional cosmetic ingredients will be of great interest.

\section{MATERIALS AND METHODS}

Detailed information is provided in the Supplementary Information.

\section{ACKNOWLEDGEMENTS}

This work was supported by an INHA UNIVERSITY Research Grant to K.K., research funds for newly appointed professors of Jeonbuk National University to S.B.L., and National Research Foundation of Korea (NRF) grants funded by the Korean government (MSIT) to K.K. (2020R1C1C1004015), S.B.L. (2020R 1C1C1014281), and C.K.K (2018R1D1A1B07050274).

\section{CONFLICTS OF INTEREST}

The authors have no conflicting interests.

\section{REFERENCES}

1. El-Domyati $M$, Attia S, Saleh F et al (2002) Intrinsic aging vs. photoaging: a comparative histopathological, immunohistochemical, and ultrastructural study of skin. Exp Dermatol 11, 398-405

2. Ganceviciene R, Liakou Al, Theodoridis A, Makrantonaki E and Zouboulis CC (2012) Skin anti-aging strategies. Dermatoendocrinol 4, 308-319

3. Rendon MI and Gaviria Jl (2005) Review of skin-lightening agents. Dermatol Surg 31, 886-889

4. Petit $L$ and Pierard GE (2003) Skin-lightening products revisited. Int J Cosmet Sci 25, 169-181

5. Kim WS, Park BS, Park SH, Kim HK and Sung JH (2009) Antiwrinkle effect of adipose-derived stem cell: activation of dermal fibroblast by secretory factors. J Dermatol Sci 53, 96-102

6. Kim J, Kang S, Kwon H, Moon H and Park MC (2019) Dual functional bioactive-peptide, AIMP1-derived peptide (AdP), for anti-aging. J Cosmet Dermatol 18, 251-257

7. Edgar S, Hopley B, Genovese L, Sibilla S, Laight D and Shute J (2018) Effects of collagen-derived bioactive peptides and natural antioxidant compounds on proliferation and matrix protein synthesis by cultured normal human dermal fibroblasts. Sci Rep 8, 10474

8. van Zuijlen PP, Ruurda JJ, van Veen HA et al (2003) Collagen morphology in human skin and scar tissue: no adaptations in response to mechanical loading at joints. Burns 29, 423-431

9. Gelse K, Poschl E and Aigner T (2003) Collagens-structure, function, and biosynthesis. Adv Drug Deliv Rev 55, 1531-1546

10. Singer AJ and Clark RA (1999) Cutaneous wound healing. N Engl J Med 341, 738-746 
11. Imanishi J, Kamiyama K, Iguchi I, Kita M, Sotozono C and Kinoshita S (2000) Growth factors: importance in wound healing and maintenance of transparency of the cornea. Prog Retin Eye Res 19, 113-129

12. Park JS, Kim JY, Cho JY, Kang JS and Yu YH (2000) Epidermal growth factor (EGF) antagonizes transforming growth factor (TGF)-beta1-induced collagen lattice contraction by human skin fibroblasts. Biol Pharm Bull 23, 15171520

13. Van Cauter E and Plat L (1996) Physiology of growth hormone secretion during sleep. J Pediatr 128, S32-37

14. Bartke A (2008) Growth hormone and aging: a challenging controversy. Clin Interv Aging 3, 659-665

15. Sonntag WE, Csiszar A, deCabo R, Ferrucci $L$ and Ungvari Z (2012) Diverse roles of growth hormone and insulin-like growth factor-1 in mammalian aging: progress and controversies. J Gerontol A Biol Sci Med Sci 67, 587-598

16. Sontakke SB, Jung JH, Piao Z and Chung HJ (2016) Orally Available Collagen Tripeptide: Enzymatic Stability, Intestinal Permeability, and Absorption of Gly-Pro-Hyp and Pro-Hyp. J Agric Food Chem 64, 7127-7133

17. Watanabe-Kamiyama $M$, Shimizu $M$, Kamiyama $S$ et al (2010) Absorption and effectiveness of orally administered low molecular weight collagen hydrolysate in rats. J Agric Food Chem 58, 835-841

18. Asserin J, Lati E, Shioya T and Prawitt J (2015) The effect of oral collagen peptide supplementation on skin moisture and the dermal collagen network: evidence from an ex vivo model and randomized, placebo-controlled clinical trials. J Cosmet Dermatol 14, 291-301

19. Inoue N, Sugihara F and Wang X (2016) Ingestion of bioactive collagen hydrolysates enhance facial skin moisture and elasticity and reduce facial ageing signs in a randomised double-blind placebo-controlled clinical study. J Sci Food Agric 96, 4077-4081

20. Konig D, Oesser S, Scharla S, Zdzieblik D and Gollhofer A (2018) Specific Collagen Peptides Improve Bone Mineral Density and Bone Markers in Postmenopausal Women-A Randomized Controlled Study. Nutrients 10, 97

21. Zdzieblik D, Oesser S, Baumstark MW, Gollhofer A and Konig D (2015) Collagen peptide supplementation in combination with resistance training improves body composition and increases muscle strength in elderly sarcopenic men: a randomised controlled trial. Br J Nutr 114, 12371245

22. Hu Z, Yang P, Zhou C, Li S and Hong P (2017) Marine Collagen Peptides from the Skin of Nile Tilapia (Oreochromis niloticus): Characterization and Wound Healing Evaluation. Mar Drugs 15, 102

23. Minh Thuy le T, Okazaki E and Osako K (2014) Isolation and characterization of acid-soluble collagen from the scales of marine fishes from Japan and Vietnam. Food Chem 149, 264-270

24. Felician FF, Xia C, Qi W and Xu H (2018) Collagen from Marine Biological Sources and Medical Applications. Chem Biodivers 15, e1700557

25. Wang B, Wang YM, Chi CF, Luo HY, Deng SG and Ma JY (2013) Isolation and characterization of collagen and antioxidant collagen peptides from scales of croceine croaker (Pseudosciaena crocea). Mar Drugs 11, 4641-4661
26. Tanaka M, Koyama $Y$ and Nomura $Y$ (2009) Effects of collagen peptide ingestion on UV-B-induced skin damage. Biosci Biotechnol Biochem 73, 930-932

27. Lee JK, Kang SI, Kim YJ et al (2016) Comparison of collagen characteristics of sea- and freshwater-rainbow trout skin. Food Sci Biotechnol 25, 131-136

28. Trojanowska M, LeRoy EC, Eckes B and Krieg T (1998) Pathogenesis of fibrosis: type 1 collagen and the skin. J Mol Med (Berl) 76, 266-274

29. Chiquet $M$, Matthisson $M$, Koch $M$, Tannheimer $M$ and Chiquet-Ehrismann R (1996) Regulation of extracellular matrix synthesis by mechanical stress. Biochem Cell Biol 74, $737-744$

30. Majora M, Wittkampf T, Schuermann B et al (2009) Functional consequences of mitochondrial DNA deletions in human skin fibroblasts: increased contractile strength in collagen lattices is due to oxidative stress-induced lysyl oxidase activity. Am J Pathol 175, 1019-1029

31. Seo WY, Kim JH, Baek DS et al (2017) Production of recombinant human procollagen type I C-terminal propeptide and establishment of a sandwich ELISA for quantification. Sci Rep 7, 15946

32. Chen CZ, Peng YX, Wang ZB et al (2009) The Scar-in-aJar: studying potential antifibrotic compounds from the epigenetic to extracellular level in a single well. $\mathrm{Br}$ J Pharmacol 158, 1196-1209

33. Adelmann-Grill BC, Wach F, Cully Z, Hein R and Krieg $T$ (1990) Chemotactic migration of normal dermal fibroblasts towards epidermal growth factor and its modulation by platelet-derived growth factor and transforming growth factor-beta. Eur J Cell Biol 51, 322-326

34. Walter MN, Wright KT, Fuller HR, MacNeil S and Johnson WE (2010) Mesenchymal stem cell-conditioned medium accelerates skin wound healing: an in vitro study of fibroblast and keratinocyte scratch assays. Exp Cell Res $316,1271-1281$

35. Quan T, Shao Y, He T, Voorhees JJ and Fisher GJ (2010) Reduced expression of connective tissue growth factor (CTGF/CCN2) mediates collagen loss in chronologically aged human skin. J Invest Dermatol 130, 415-424

36. Seite S, Zucchi H, Septier D, Igondjo-Tchen S, Senni K and Godeau G (2006) Elastin changes during chronological and photo-ageing: the important role of lysozyme. J Eur Acad Dermatol Venereol 20, 980-987

37. Liu Z, Li Y, Song $\mathrm{H}$ et al (2019) Collagen peptides promote photoaging skin cell repair by activating the TGFbeta/Smad pathway and depressing collagen degradation. Food Funct 10, 6121-6134

38. Fisher GJ, Choi HC, Bata-Csorgo Z et al (2001) Ultraviolet irradiation increases matrix metalloproteinase-8 protein in human skin in vivo. J Invest Dermatol 117, 219-226

39. Sugimoto K, Nakamura T, Tokunaga T et al (2018) Matrix metalloproteinase promotes elastic fiber degradation in ligamentum flavum degeneration. PLoS One 13, e0200872

40. Chung JH, Seo JY, Choi HR et al (2001) Modulation of skin collagen metabolism in aged and photoaged human skin in vivo. J Invest Dermatol 117, 1218-1224

41. Martinotti S and Ranzato E (2020) Scratch Wound Healing Assay. Methods Mol Biol 2109, 225-229 